\title{
Resolution of neonatal posthemorrhagic ventricular dilation coincident with patent ductus arteriosus ligation: case report
}

\author{
Erik B. Vanstrum, BA, ${ }^{1}$ Matthew T. Borzage, PhD, ${ }^{3}$ Jason K. Chu, MD, MSc, ${ }^{1,2}$ Shuo Wang, MD, ${ }^{4}$ \\ Nolan Rea, BS, ${ }^{2}$ J. Gordon McComb, MD, ${ }^{1,2}$ Mark D. Krieger, MD, ${ }^{1,2}$ and \\ Peter A. Chiarelli, MD, DPhil ${ }^{1,2}$ \\ ${ }^{1}$ Keck School of Medicine of University of Southern California, Los Angeles; and Divisions of ${ }^{2}$ Neurosurgery, ${ }^{3}$ Neonatology, and \\ ${ }^{4}$ Cardiology, Children's Hospital Los Angeles, California
}

\begin{abstract}
Preterm infants commonly present with a hemodynamically significant patent ductus arteriosus (hsPDA). The authors describe the case of a preterm infant with posthemorrhagic ventricular dilation, which resolved in a temporally coincident fashion to repair of hsPDA. The presence of a PDA with left-to-right shunting was confirmed at birth on echocardiogram and was unresponsive to repeated medical intervention. Initial cranial ultrasound revealed periventricular-intraventricular hemorrhage. Follow-up serial ultrasound showed resolving intraventricular hemorrhage and progressive bilateral hydrocephalus. At 5 weeks, the ductus was ligated with the goal of improving hemodynamic stability prior to CSF diversion. However, neurosurgical intervention was not required due to improvement of ventriculomegaly occurring immediately after PDA ligation. No further ventricular dilation was observed at the 6-month follow-up.

Systemic venous flow disruption and abnormal patterns of cerebral blood circulation have been previously associated with hsPDA. Systemic hemodynamic change has been reported to follow hsPDA ligation, although association with ventricular normalization has not. This case suggests that the unstable hemodynamic environment due to left-to-right shunting may also impede CSF outflow and contribute to ventriculomegaly. The authors review the literature surrounding pressure transmission between a PDA and the cerebral vessels and present a mechanism by which PDA may contribute to posthemorrhagic ventricular dilation.
\end{abstract}

https://thejns.org/doi/abs/10.3171/2020.3.PEDS19694

KEYWORDS hydrocephalus; patent ductus arteriosus; intraventricular hemorrhage; premature infant; posthemorrhagic ventricular dilation; neonatal

$\mathrm{T}$ HE ductus arteriosus facilitates blood flow from the pulmonary artery (PA) to the aorta during gestation. This vascular pathway typically closes within hours after birth, but can remain patent, especially in infants of decreased gestational age. ${ }^{1,2}$ An estimated $20 \%$ of premature neonates are born with a patent ductus arteriosus (PDA). ${ }^{3-5}$ A hemodynamically significant PDA (hsPDA) can be associated with harmful alterations in vascular flow, volume, and pressure that are experienced by the brain. ${ }^{6}$ Although there is not a consensus on defining a threshold for hsPDA, Shepherd and Noori recently suggested criteria for objective measures of PDA flow (e.g., ductal diameter, shunt pattern) and clinical factors (e.g., prematurity, periventricular-intraventricular hemorrhage $[\mathrm{PIVH}])$ that are associated with hemodynamic significance. ${ }^{6}$ The abnormal vascular effects of left-to-right cardiac shunting can increase the risk of neonatal PIVH due to rupture of fragile germinal matrix microvasculature. ${ }^{7}$ Circulatory changes attributed to hsPDA that correlate with IVH severity include retrograde flow in cerebral arteries, ${ }^{8}$ decreased right ventricular output, ${ }^{9}$ decreased diastolic blood pressure, ${ }^{7,10}$ and decreased superior vena cava (SVC) flow rate. ${ }^{11}$ Infants with hsPDA can have elevated central venous pressure (CVP) ${ }^{7,12}$ and may demonstrate an

ABBREVIATIONS CVP = central venous pressure; FOHR = frontal-occipital horn ratio; FTHR = frontal-temporal horn ratio; hsPDA = hemodynamically significant PDA; $\mathrm{OFC}=$ occipital frontal circumference; $\mathrm{PA}=$ pulmonary artery; $\mathrm{PDA}=$ patent ductus arteriosus; $\mathrm{PHVD}=$ posthemorrhagic ventricular dilation; $\mathrm{PIVH}=$ periventricular-intraventricular hemorrhage; SVC = superior vena cava; VLBW = very low birth weight; VSGS = ventriculosubgaleal shunt.

SUBMITTED November 20, 2019. ACCEPTED March 30, 2020.

INCLUDE WHEN CITING Published online May 22, 2020; DOI: 10.3171/2020.3.PEDS19694. 
inverse trend between PDA diameter and cerebral oxygen saturation. ${ }^{13,14}$ There is also evidence that hsPDA may have a negative impact on neurodevelopment. ${ }^{15-17}$

Increases in PDA size correlate with increased rates of adverse sequelae (e.g., pulmonary hypertension, PIVH) $)^{9,18}$ and are important factors in predicting future ductus patency and the need for treatment. ${ }^{19}$ Treatment strategies for hsPDA include conservative monitoring for spontaneous closure, pharmaceutical intervention, endovascular coiling, and surgical ligation. ${ }^{20,21}$ Ligation is generally reserved for hsPDA unresponsive to conservative or medical management. ${ }^{22}$ In the US, there is considerable variation in PDA diagnosis and treatment by geographic region, with ligation rates in PDA-bearing infants ranging from $4 \%$ to $28 \% .^{5}$ In preterm infants overall, the incidence of PIVH is $25 \%-30 \%$, with grade III or IV hemorrhage comprising approximately one-third of bleeding events. ${ }^{3,23}$ Nearly $75 \%$ of babies with grade III and IV PIVH demonstrate persistent posthemorrhagic ventricular dilation (PHVD), and $75 \%$ of these neonates with PHVD progress to a formal diagnosis of hydrocephalus requiring CSF diversion. ${ }^{24}$

In this work, we report the spontaneous resolution of PIVH-associated ventriculomegaly immediately following hsPDA ligation. Within the body of literature on neonatal ventriculomegaly and PIVH-associated hydrocephalus, a rigorous examination regarding the time course of ventricular enlargement is lacking. Additional mechanistic insight is required regarding the origins of neonatal ventricular dilation and the resolution of this phenomenon when it occurs. ${ }^{25}$ To our knowledge, the reversal of progressive PHVD has an unreported association with hsPDA ligation. Whereas the pathway for arterial pressure transmission to the systemic venous circulation and consequent venous flow disruption in the brain has been well established in hsPDA, the link with neonatal ventriculomegaly has not been explored. We believe this work will serve to facilitate further targeted investigation, and we propose a mechanism through which such an effect may occur.

\section{Case Report}

History and Examination

The patient was male, born weighing $615 \mathrm{~g}$ at 25 weeks' gestation to a 35 -year-old mother $(\mathrm{G} 2 \mathrm{P} 1)$ via repeat cesarean section due to fetal breech position. The pregnancy was complicated by superimposed preeclampsia with severe features, and fetal cardiac decelerations. The boy was admitted to the neonatal intensive care unit for management of prematurity, extremely low birth weight, and bronchopulmonary dysplasia. He received multiple courses of dopamine and stress-dose hydrocortisone for blood pressure support.

High-frequency oscillatory ventilation was used for worsening respiratory acidosis and pulmonary edema secondary to suspected PDA. The presence of a PDA with significant left-to-right cardiovascular shunting was confirmed on echocardiogram. Pharmaceutical intervention was implemented with 2 rounds of acetaminophen, followed by 2 rounds of indomethacin. Serial echocardiograms demonstrated continued patency of the ductus despite medical intervention, and further revealed a small

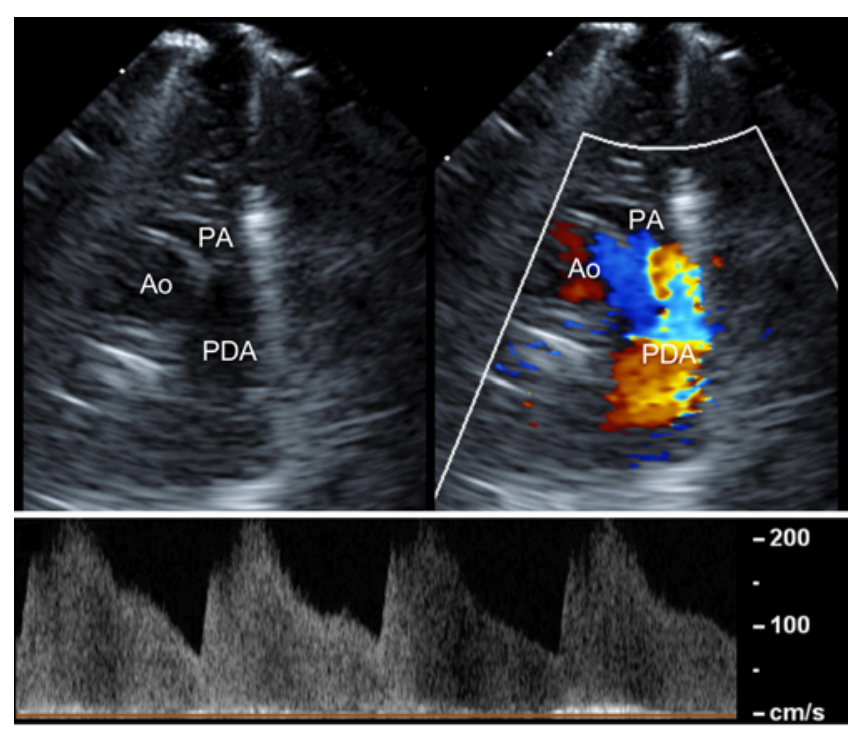

FIG. 1. Preoperative PDA echocardiogram images and Doppler flow. Upper: Representative 2D imaging of the PDA with and without Doppler color. Lower: Doppler tracing of PDA blood flow showing pulsatile leftto-right shunt pattern. Ao $=$ aorta. Figure is available in color online only.

patent foramen ovale with normal right ventricle size, a mildly dilated left ventricle, and physiological tricuspid valve insufficiency.

Initial cranial ultrasound showed enlarged lateral, third, and fourth ventricles with IVH and bilateral subependymal hemorrhage consistent with grade III PIVH. Followup ultrasounds revealed progressive bilateral ventricular enlargement with resolving IVH and no new bleeding. The patient had a progressively increasing frontal-occipital horn ratio (FOHR) above 0.55 , progressively full-but-soft fontanelle, and splayed sutures. Multidisciplinary discussion with cardiac and neonatal intensive care teams encouraged improved hemodynamic stability prior to a cranial temporizing surgery (e.g., ventriculosubgaleal shunt [VSGS]), with the consensus that PDA ligation might contribute to improved cardiopulmonary function.

\section{Operation, Postoperative Imaging, and Follow-Up}

At week 3 of life, the PDA measured $1.7 \mathrm{~mm}$ in diameter on echocardiograms, with a pulsatile, left-to-right shunting pattern (Fig. 1). Additional measurements suggestive of hemodynamic significance included a left atrium/aortic root ratio of 1.7, a left PA end diastolic velocity of $16 \mathrm{~cm} / \mathrm{sec}$, and a left ventricle output of $428 \mathrm{ml} / \mathrm{kg} / \mathrm{min} .{ }^{6}$ No reversal of flow was noted in the descending aorta. On day 35 of life, the patient underwent surgical ligation of the ductus arteriosus via a posterolateral fourth interspace thoracotomy. Postoperative echocardiograms revealed ductal hemostasis, a widely patent aortic arch, and normal cardiac ventricular function.

The plans for cranial temporizing surgery were influenced by coincident changes in postligation measurements of ventricular size. Figure 2 displays data from all cranial ultrasounds obtained between weeks 1 and 15 of life, including calculated measures of FOHR (Fig. 2A), frontaltemporal horn ratio (FTHR; Fig. 2B), temporal horn width 

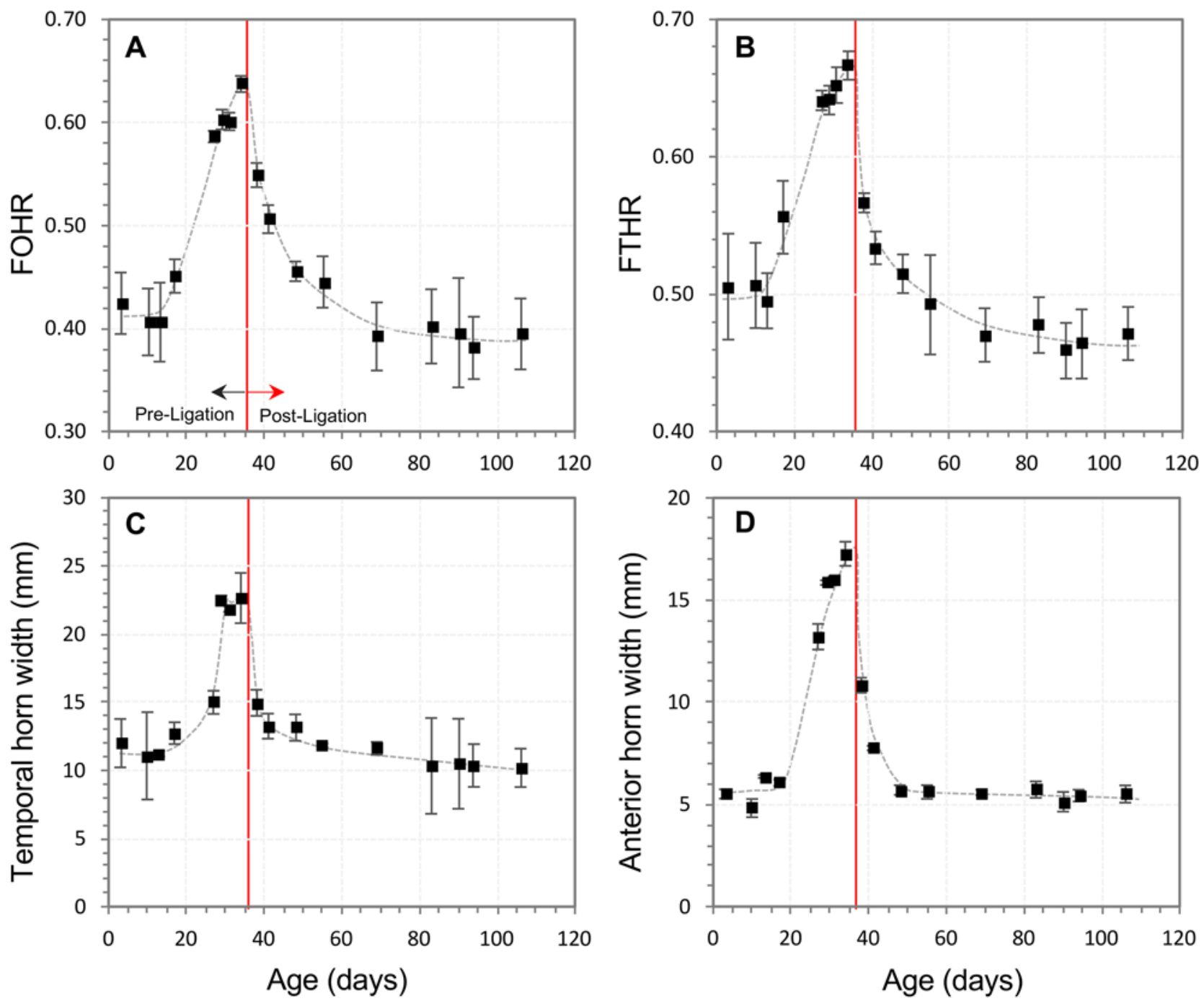

FIG. 2. Graphs showing time course data obtained using 4 techniques of ventricular size estimation. Cranial ultrasound images were used to calculate (A) FOHR, (B) FTHR, (C) temporal horn width, and (D) maximum bilateral frontal horn width over time. Ventricle measurements were made in triplicate, once each by 3 individuals, with error estimates between measurements displayed as $\pm \mathrm{SD}$. The day of hsPDA ligation is indicated by the vertical red line. Figure is available in color online only.

(Fig. 2C), and anterior horn width (Fig. 2D), along with the timing of PDA ligation (red line). Representative cranial ultrasound coronal sections are supplied in Fig. 3. Serial ultrasound images showed a transition between worsening and improvement of ventriculomegaly occurring immediately at the time of PDA ligation. There was no substantial change in the distribution of intraventricular clot during this time frame. As a result, neurosurgical intervention was averted. Immediately prior to ligation, the occipital frontal circumference (OFC) began to increase past growth curves, above the 10th percentile, to reflect the ventricular size change (Fig. 4). This upswing normalizes thereafter to a trajectory matching the 5 th percentile. This may have been due to difference in OFC measuring techniques between individuals.

At 3 months of age, echocardiogram revealed normal cardiac biventricular size and function, unchanged mild patent foramen ovale, a patent aortic arch, and stasis of flow through the prior PDA site. Cranial ventricular size and head circumference remained stable, and no signs of transient bradycardia or apnea were present (Fig. 4). The fontanelle was soft and sunken. No temporizing measures for CSF extraction were performed throughout the patient's clinical course. No further ventricular dilation was observed at 6 months of follow-up, and no shunt has been required.

\section{Discussion}

In this work we present a case of ventriculomegaly resolution that occurred in the absence of intracranial intervention, immediately following hsPDA closure. According to recently suggested guidelines to define the hemodynamic significance of PDA flow, this case meets 4 of the 6 objective criteria (size $>1.5 \mathrm{~mm}$ at gestational age $<26$ weeks, pulsatile shunt pattern, left atrium/aortic 

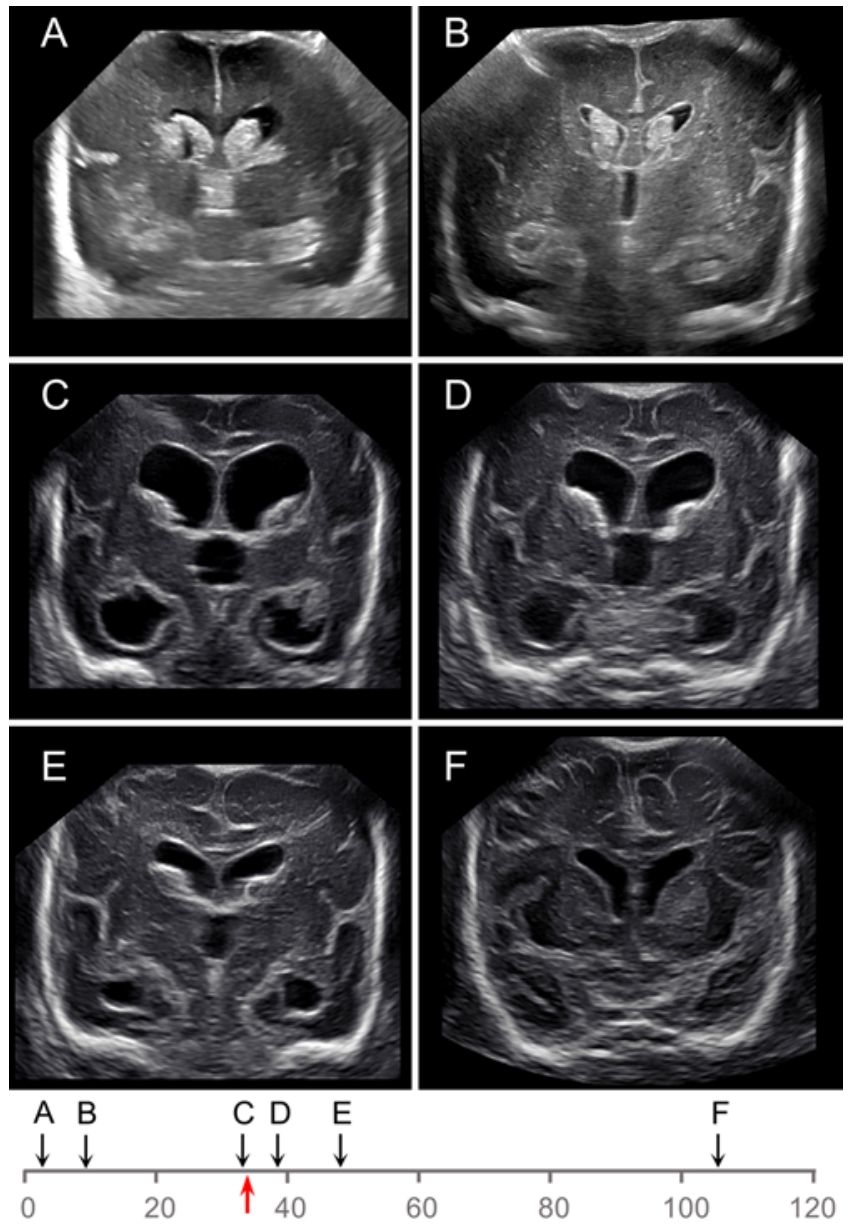

Age (days)

FIG. 3. Representative cranial ultrasound images. Images are displayed from days of life $3,10,34,38,48$, and 106 and correspond to arrows on the timeline below. The day of hsPDA ligation is indicated by the red arrow. Figure is available in color online only.

root ratio $>1.4$, and left ventricle output $>300 \mathrm{ml} / \mathrm{kg} / \mathrm{min}$ ) with 2 additional contributing clinical factors (prematurity, IVH). ${ }^{6}$ Figure 5 provides a schematic representation for possible vascular feedback between the cardiac and intracranial compartments, using known systemic hemodynamic consequences of hsPDA to support a plausible connection with ventriculomegaly. Left-to-right shunting of blood through a PDA allows mixing of the high-pressure systemic circulation and low-pressure pulmonary circulation. ${ }^{26}$ Infants with hsPDA have decreased right ventricle output, increased SVC resistance, and decreased cerebral oxygen saturation. ${ }^{9,11,13}$ Prior reports suggest that PDAassociated preloading of the right heart contributes to venous congestion within the dural venous sinuses. ${ }^{13}$ Very low birth weight (VLBW) infants often have near- or subatmospheric intracranial pressure, making small changes in systemic circulation likely to influence cerebral venous hemodynamics..$^{27,28}$ Elevated CVP and impaired venous outflow from the sinus represent compelling potential sources of theoretical impairment for CSF egress (Fig. 5).

The data supplied here convey a correlation of PDA clo-
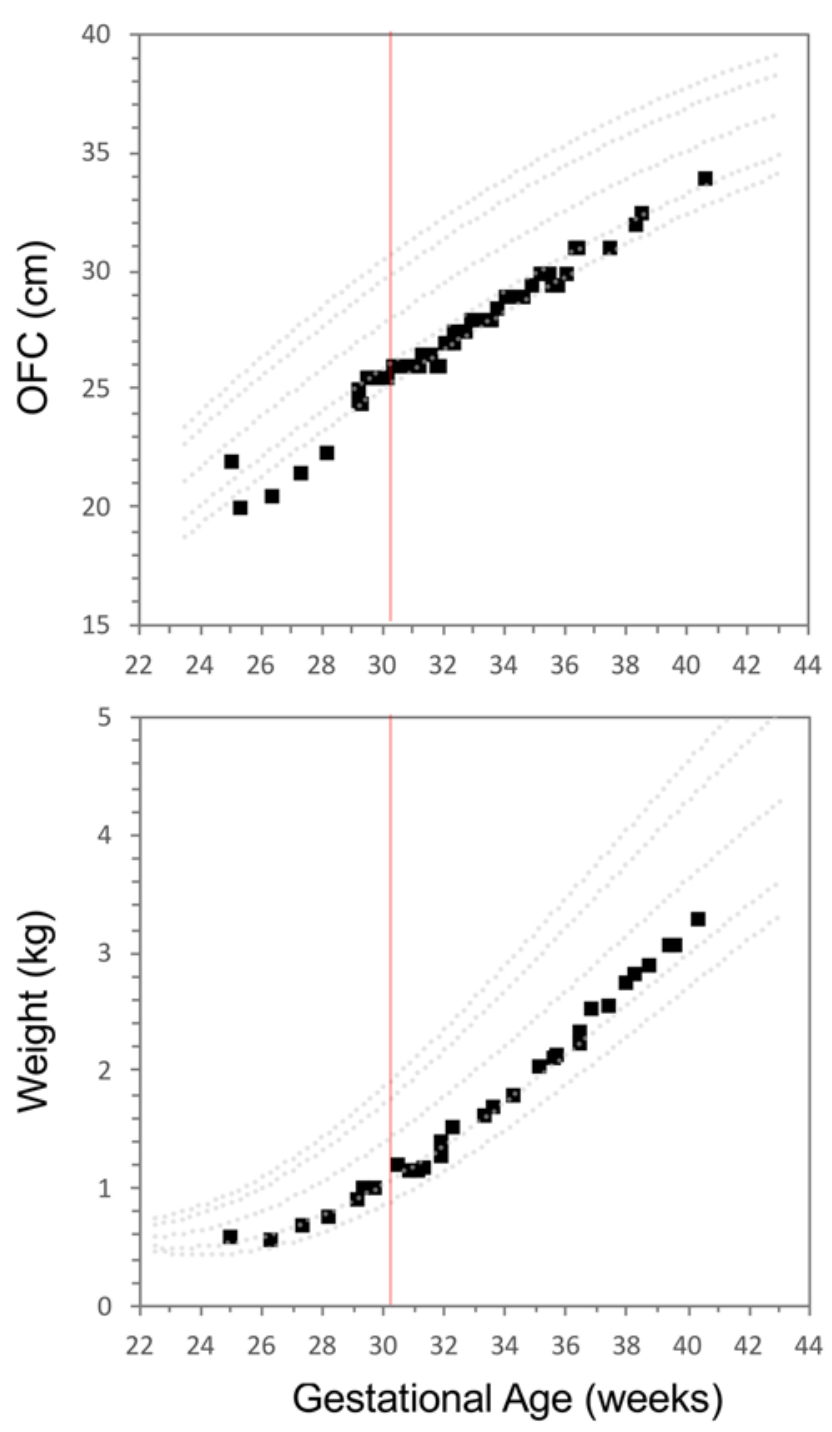

FIG. 4. OFC and weight change over time. OFC (cm) and weight $(\mathrm{kg})$ were plotted along a Fenton growth chart for premature infants with gestational age (weeks) as the $\mathrm{x}$-axis. From top to bottom, dashed lines indicate the 97 th, 90th, 50th, 10th, and 3rd percentiles. Figure is available in color online only.

sure with ventricular size/OFC normalization (Figs. 2-4) and represent the first documentation of this temporal relationship in the literature. The results must be interpreted with caution and should not be viewed as proof of a link between hsPDA, cerebral hemodynamics, and ventricular outflow. Although hydrocephalus is a well-documented consequence of PIVH, ${ }^{3,23}$ our case suggests that the unstable hemodynamic environment due to left-to-right shunting may also impede CSF outflow and, in addition to an underlying degree of IVH, may contribute mechanistically to ventriculomegaly. This relationship may not have been previously identified due to the nationwide variation in PDA diagnosis and treatment strategy, ${ }^{5}$ or to the lack of a definition surrounding PDA hemodynamic significance. ${ }^{6}$

It has been previously shown that a systemic hemodynamic change can immediately follow hsPDA ligation. Bissonnette and Benson detected both acute decline in 


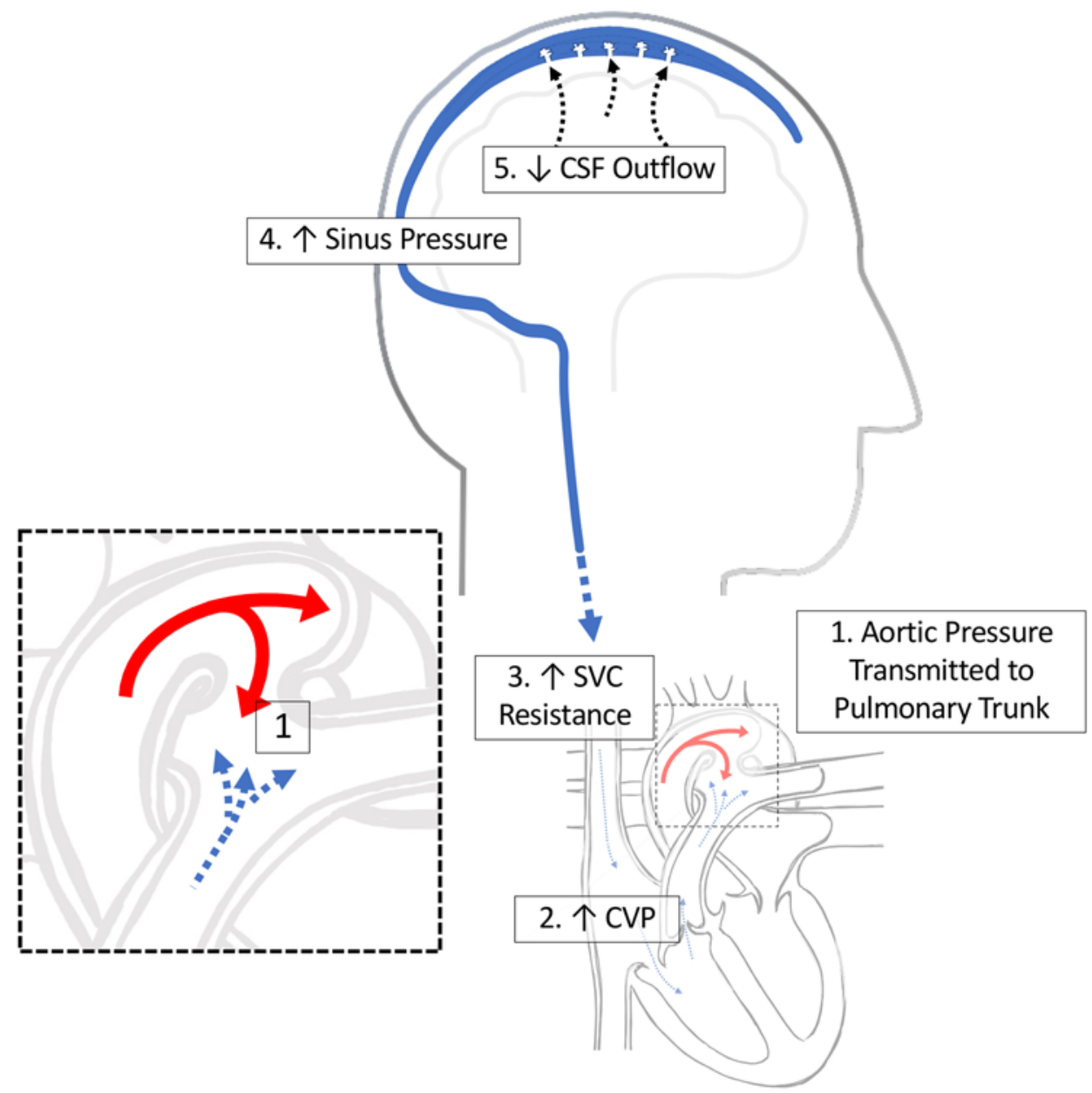

FIG. 5. Proposed mechanism to explain hydrocephalus in the context of hsPDA. Left-to-right shunting via hsPDA diverts blood from the systemic arterial circulation to the PA. Preloading of the right heart can result in increased CVP and SVC resistance, leading to venous congestion in the brain and reduced CSF outflow. Dashed lines indicate increased fluid resistance. Figure is available in color online only.

CVP and increase in diastolic cerebral artery velocity during surgical PDA ligation, ${ }^{7}$ a finding supported by others. ${ }^{10,29}$ In an investigation of the natural history of VLBW infants with posthemorrhagic ventriculomegaly, $38 \%$ had arrest of cerebral ventricular enlargement and did not require treatment. ${ }^{23}$ Thus a percentage of PVHD cases spontaneously resolve. In a multiinstitutional study of premature newborns with IVH grade III or IV, $19 \%$ of infants (28/145) did not require any temporization. ${ }^{24}$ Unfortunately, a majority of literature reports have not supplied time course details of ventricle size change, which would help to support or deny this relationship. One illustrative case report by Allan et al. suggests that resolution of ventricular dilation without intervention can occur over a 2- to 4 -week time frame, a longer time frame than seen here. ${ }^{30}$ The use of temporizing measures (i.e., lumbar puncture, fontanelle tap, external ventricular drainage, ventricular reservoir, VSGS) for severe PHVD further confounds interpretation of trends in the literature. ${ }^{31-33}$ Resolution of ventriculomegaly after temporizing interventions may be attributed to the intervention itself-by reducing CSF protein content, red blood cells, or inflammatory factors-or to the time delay and systemic hemodynamic changes afforded by the intervention. Kaiser and Whitelaw provide a case similar to ours, in which a VLBW infant of 28 weeks' gestation with PHVD experienced a rapid decline in ventricular width over the course of a day. ${ }^{32}$ This decline followed 4 serial lumbar punctures performed 1 week prior. It is interesting to note that this patient's course was complicated by a PDA, although a link to PDA extent, treatment, monitoring, or resolution was not explored. Further research is required to examine the physiological nature and timing of spontaneous ventriculomegaly resolution.

The mechanism by which ventriculomegaly may appear and resolve following IVH has been underexplored to date. ${ }^{25}$ A number of theories exist for posthemorrhagic CSF outflow disruption, including 1) temporary thrombotic or proteinaceous obstruction of CSF outflow through foramina or arachnoid granulations; 2) inflammatorymediated chemical arachnoiditis; 3) posthemorrhagic distortion of normal CSF and/or vascular pressure gradients; and 4) impaired lymphatic CSF resorption..$^{25,34-36}$ Whereas prior models accepted the arachnoid granulation as a primary site of CSF outflow, current understanding places 
additional importance on interstitial CSF flow. ${ }^{36,37}$ In premature infants, the arachnoid villi and granulations are in development, suggesting other routes of CSF drainage. ${ }^{38,39}$ In animal models, venous drainage of CSF via granulations serves as an auxiliary system, with increases in flow at times of elevated intracranial pressure. ${ }^{40}$

The patient described in this report would probably have undergone placement of a VSGS prior to PDA ligation had he been hemodynamically stable. The preceding PDA ligation allowed visualization of ventricular size reduction in the absence of cranial temporizing intervention. The proportion of infants who demonstrate resolution of ventriculomegaly or a change in cerebral hemodynamics after PDA closure is unknown. This question may be addressed in the future by rigorous collection of hemodynamic data (e.g., cranial arterial/venous Doppler ultrasound, echocardiogram, systemic venous pressure) and modern neuroimaging data (e.g., structural MRI, CSF flow sequences, MR perfusion) during a period of transient ventriculomegaly. Few studies of hydrocephalus include data on hsPDA severity or closure time. This report of hsPDA ligation and PHVD highlights the potential connection between systemic hemodynamics and CSF flow in neonates, and provides new data from a single patient regarding the time course along which ventriculomegaly may improve. Furthermore, this work suggests a role for future prospective data collection regarding PDA, intracranial hemodynamics, and ventricular size.

\section{Acknowledgments}

A Summer Oncology Research Fellowship, Children's Hospital Los Angeles, was awarded to Erik Vanstrum.

\section{References}

1. Noori S, McCoy M, Friedlich P, et al. Failure of ductus arteriosus closure is associated with increased mortality in preterm infants. Pediatrics. 2009;123(1):e138-e144.

2. Tauzin L, Joubert C, Noel A-C, et al. Effect of persistent patent ductus arteriosus on mortality and morbidity in very lowbirthweight infants. Acta Paediatr. 2012;101(4):419-423.

3. Christian EA, Jin DL, Attenello F, et al. Trends in hospitalization of preterm infants with intraventricular hemorrhage and hydrocephalus in the United States, 2000-2010. J Neurosurg Pediatr. 2016;17(3):260-269.

4. Ferré C, Callaghan W, Olson C, et al. Effects of maternal age and age-specific preterm birth rates on overall preterm birth rates-United States, 2007 and 2014. MMWR Morb Mortal Wkly Rep. 2016;65(43):1181-1184.

5. Weinberg JG, Evans FJ, Burns KM, et al. Surgical ligation of patent ductus arteriosus in premature infants: trends and practice variation. Cardiol Young. 2016;26(6):1107-1114.

6. Shepherd JL, Noori S. What is a hemodynamically significant PDA in preterm infants? Congenit Heart Dis. 2019;14(1):21-26.

7. Bissonnette B, Benson LN. Closure of persistently patent arterial duct and its impact on cerebral circulatory haemodynamics in children. Can J Anaesth. 1998;45(3):199-205.

8. Martin CG, Snider AR, Katz SM, et al. Abnormal cerebral blood flow patterns in preterm infants with a large patent ductus arteriosus. J Pediatr. 1982;101(4):587-593.

9. Evans N, Kluckow M. Early ductal shunting and intraventricular haemorrhage in ventilated preterm infants. Arch Dis Child Fetal Neonatal Ed. 1996;75(3):F183-F186.

10. Ratner I, Perelmuter B, Toews W, Whitfield J. Association of low systolic and diastolic blood pressure with significant patent ductus arteriosus in the very low birth weight infant. Crit Care Med. 1985;13(6):497-500.

11. Kluckow M, Evans N. Low superior vena cava flow and intraventricular haemorrhage in preterm infants. Arch Dis Child Fetal Neonatal Ed. 2000;82(3):F188-F194.

12. Siassi B, Wu PY, Li RK, Mondanlou H. Central venous pressure in preterm infants. Biol Neonate. 1980;37(5-6):285-290.

13. Dix L, Molenschot M, Breur J, et al. Cerebral oxygenation and echocardiographic parameters in preterm neonates with a patent ductus arteriosus: an observational study. Arch Dis Child Fetal Neonatal Ed. 2016;101(6):F520-F526.

14. Lemmers PMA, Toet MC, van Bel F. Impact of patent ductus arteriosus and subsequent therapy with indomethacin on cerebral oxygenation in preterm infants. Pediatrics. 2008;121(1):142-147.

15. Cheong JL, Doyle LW, Burnett AC, et al. Association between moderate and late preterm birth and neurodevelopment and social-emotional development at age 2 years. JAMA Pediatr. 2017;171(4):e164805.

16. Collins RT II, Lyle RE, Rettiganti M, et al. Long-term neurodevelopment of low-birthweight, preterm infants with patent ductus arteriosus. J Pediatr. 2018;203:170-176.e1.

17. Janz-Robinson EM, Badawi N, Walker K, et al. Neurodevelopmental outcomes of premature infants treated for patent ductus arteriosus: a population-based cohort study. J Pediatr. 2015;167(5):1025-1032.e3.

18. Jim W-T, Chiu N-C, Chen M-R, et al. Cerebral hemodynamic change and intraventricular hemorrhage in very low birth weight infants with patent ductus arteriosus. Ultrasound Med Biol. 2005;31(2):197-202.

19. Yum SK, Moon C-J, Youn Y-A, et al. Echocardiographic assessment of patent ductus arteriosus in very low birthweight infants over time: prospective observational study. J Matern Fetal Neonatal Med. 2018;31(2):164-172.

20. Dice JE, Bhatia J. Patent ductus arteriosus: an overview. $J$ Pediatr Pharmacol Ther. 2007;12(3):138-146.

21. Rothman A, Lucas VW, Sklansky MS, et al. Percutaneous coil occlusion of patent ductus arteriosus. J Pediatr. 1997;130(3):447-454.

22. Van Overmeire B, Chemtob S. The pharmacologic closure of the patent ductus arteriosus. Semin Fetal Neonatal Med. 2005;10(2):177-184.

23. Murphy BP, Inder TE, Rooks V, et al. Posthaemorrhagic ventricular dilatation in the premature infant: natural history and predictors of outcome. Arch Dis Child Fetal Neonatal Ed. 2002;87(1):F37-F41.

24. Wellons JC III, Shannon CN, Holubkov R, et al. Shunting outcomes in posthemorrhagic hydrocephalus: results of a Hydrocephalus Clinical Research Network prospective cohort study. J Neurosurg Pediatr. 2017;20(1):19-29.

25. Koschnitzky JE, Keep RF, Limbrick DD Jr, et al. Opportunities in posthemorrhagic hydrocephalus research: outcomes of the Hydrocephalus Association Posthemorrhagic Hydrocephalus Workshop. Fluids Barriers CNS. 2018;15(1):11.

26. Spach MS, Serwer GA, Anderson PA, et al. Pulsatile aortopulmonary pressure-flow dynamics of patent ductus arteriosus in patients with various hemodynamic states. Circulation. 1980;61(1):110-122.

27. Welch K. The intracranial pressure in infants. J Neurosurg. 1980;52(5):693-699.

28. Yada K, Nakagawa Y, Tsuru M. Site and mechanism of vascular stenosis under increased intracranial pressure-intracranial venous pressure regulation mechanism. In: Lundberg $\mathrm{N}$, Pontén U, Brock M, eds. Intracranial Pressure II. Springer; 1975:427-432.

29. Swarup J, Baker RW, Brozanski BS, Yanowitz TD. Asymmetry of cerebral blood flow velocity in low birth weight infants. Biol Neonate. 2005;87(3):145-151. 
30. Allan WC, Holt PJ, Sawyer LR, et al. Ventricular dilation after neonatal periventricular-intraventricular hemorrhage. Natural history and therapeutic implications. Am J Dis Child. 1982;136(7):589-593.

31. Goldstein GW, Chaplin ER, Maitland J, Norman D. Transient hydrocephalus in premature infants: treatment by lumbar punctures. Lancet. 1976;1(7958):512-514.

32. Kaiser AM, Whitelaw AG. Cerebrospinal fluid pressure during post haemorrhagic ventricular dilatation in newborn infants. Arch Dis Child. 1985;60(10):920-924.

33. Papile L-A, Burstein J, Burstein R, et al. Posthemorrhagic hydrocephalus in low-birth-weight infants: treatment by serial lumbar punctures. J Pediatr. 1980;97(2):273-277.

34. McCrea HJ, Ment LR. The diagnosis, management, and postnatal prevention of intraventricular hemorrhage in the preterm neonate. Clin Perinatol. 2008;35(4):777-792, vii.

35. Nagra G, Wagshul ME, Rashid S, et al. Elevated CSF outflow resistance associated with impaired lymphatic CSF absorption in a rat model of kaolin-induced communicating hydrocephalus. Cerebrospinal Fluid Res. 2010;7(1):4.

36. Strahle J, Garton HJL, Maher CO, et al. Mechanisms of hydrocephalus after neonatal and adult intraventricular hemorrhage. Transl Stroke Res. 2012;3(suppl 1):25-38.

37. Bothwell SW, Janigro D, Patabendige A. Cerebrospinal fluid dynamics and intracranial pressure elevation in neurological diseases. Fluids Barriers CNS. 2019;16(1):9.

38. Johnston M, Zakharov A, Papaiconomou C, et al. Evidence of connections between cerebrospinal fluid and nasal lymphatic vessels in humans, non-human primates and other mammalian species. Cerebrospinal Fluid Res. 2004;1(1):2.
39. Osaka K, Handa H, Matsumoto S, Yasuda M. Development of the cerebrospinal fluid pathway in the normal and abnormal human embryos. Childs Brain. 1980;6(1):26-38.

40. Papaiconomou C, Zakharov A, Azizi N, et al. Reassessment of the pathways responsible for cerebrospinal fluid absorption in the neonate. Childs Nerv Syst. 2004;20(1):29-36.

\section{Disclosures}

The authors report no conflict of interest concerning the materials or methods used in this study or the findings specified in this paper.

\section{Author Contributions}

Conception and design: Chiarelli, Vanstrum. Acquisition of data: Chiarelli, Vanstrum, Wang, Rea. Analysis and interpretation of data: all authors. Drafting the article: Chiarelli, Vanstrum, Borzage, Chu, Rea. Critically revising the article: Chiarelli, Vanstrum, Borzage, Chu, McComb, Krieger. Reviewed submitted version of manuscript: Chiarelli, Vanstrum, Borzage, Chu, Wang, McComb, Krieger. Approved the final version of the manuscript on behalf of all authors: Chiarelli. Study supervision: Chiarelli.

\section{Correspondence}

Peter A. Chiarelli: Children's Hospital Los Angeles, CA. pchiarelli@chla.usc.edu. 